\title{
PROGNOSTIC SIGNIFICANCE OF A COMPREHENSIVE HISTOLOGIC EVALUATION OF RETICULIN FIBROSIS, COLLAGEN DEPOSITION AND OSTEOSCLEROSIS IN PRIMARY MYELOFIBROSIS PATIENTS
}

Running title: Stromal changes in primary myelofibrosis.

Authors: Umberto Gianelli ${ }^{1}$, Stefano Fiori ${ }^{1}$, Daniele Cattaneo², Anna Bossi ${ }^{3}$, Ivan Cortinovis ${ }^{3}$, Arturo Bonometti ${ }^{1}$, Giulia Ercoli1 ${ }^{1}$ Cristina Bucelli2, Nicola Orofino², Gaetano Bulfamante ${ }^{4}$, Alessandra lurlo².

\section{Affiliations:}

${ }^{1}$ Division of Pathology, Department of Pathophysiology and Transplantation, University of Milan, and IRCCS Ca' Granda - Maggiore Policlinico Hospital Foundation, Milan, Italy.

2 Hematology Division, IRCCS Ca' Granda - Maggiore Policlinico Hospital Foundation, and Department of Oncology and Hemato-oncology, University of Milan, Milan, Italy.

${ }^{3}$ Laboratory G.A. Maccacaro, Department of Clinical Sciences and Community Health, University of Milan, Milan, Italy.

${ }^{4}$ Division of Pathology, Department of Health Sciences, University of Milan and San Paolo Hospital, Milan, Italy.

This article has been accepted for publication and undergone full peer review but has not been through the copyediting, typesetting, pagination and proofreading process, which may lead to differences between this version and the Version of Record. Please cite this article as doi: 10.1111/his.13309

This article is protected by copyright. All rights reserved. 


\section{Corresponding author:}

Umberto Gianelli, MD,

Division of Pathology,

Department of Pathophysiology and Transplantation, University of Milan,

IRCCS Ca' Granda - Maggiore Policlinico Hospital Foundation,

Via Francesco Sforza 35, 20122 Milan, Italy.

Phone number: (+39) 0255035875

Fax number: $(+39) 0255034608$

e-mail: umberto.gianelli@unimi.it

Disclosure/Conflict of interest: Authors have no disclosure or conflicts of interest to report.

\section{ABSTRACT}

Aims: to evaluate whether a comprehensive histological evaluation of reticulin fibrosis, collagen deposition and osteosclerosis in bone marrow trephine biopsies (BMBs) of primary myelofibrosis (PMF) patients may have prognostic implications.

Methods: reticulin fibrosis, collagen deposition and osteosclerosis were graded from 0 to 3 in a series of 122 base-line BMBs. Then, we assigned to each case a comprehensive score (RCO score, ranging from 0 to 9 ) that allowed us to distinguish two groups of patients, with low-grade (RCO score 0-4) and high-grade (RCO score 5-9) stromal changes.

Results and discussion: of 122 patients, 88 displayed a low-grade and 34 a high-grade RCO score. The latter was more frequently associated with anemia, thrombocytopenia, peripheral blood blasts and increased lactate dehydrogenase levels. RCO score resulted strictly correlated with overall mortality $(p=0.013)$ and International Prognostic Scoring System (IPSS) risk categories, and was able to discriminate the overall survival of both low- and high-grade patients (Log-Rank test: $p<0.001$ ). Moreover, it proved to be more accurate than the European Consensus on grading of bone marrow fibrosis (ECGMF grade) in identifying high-risk patients with poor prognosis.

This article is protected by copyright. All rights reserved. 
Finally, a combined analysis of RCO scores and IPSS risk categories in an integrated clinicalpathological evaluation was able to increase the positive predictive value (PPV) for mortality in high-risk patients.

Conclusion: the comprehensive RCO score, obtained by histological evaluation of reticulin fibrosis, collagen deposition and osteosclerosis, resulted prognostically significant, more accurate than ECGMF grade in identifying high-risk patients, and improved PPV when applied in addition to IPSS.

Key words: primary myelofibrosis; bone marrow morphology; reticulin fibrosis; collagen deposition; osteosclerosis.

\section{INTRODUCTION}

Reticulin fibers are a physiological component of bone marrow stroma, and can be appreciated as a loose meshwork in histological sections stained with silver impregnation. Several reactive and neoplastic conditions elicit an increase of the normal meshwork, and an advanced degree of reticulin fibrosis is often accompanied by other stromal changes, such as collagen deposition, osteosclerosis and microvessel proliferation. These changes are not physiologically found in bone marrow, exception made for sporadic perivascular collagen fibers. The pathogenesis of bone marrow stromal changes is unclear, but evidences suggest that reticulin fibrosis and collagen deposition may differ in terms of reversibility and clinical implications $[1,2]$.

Attempts to estimate the grade of bone marrow fibrosis have been carried out since the first grading system in 1971 [3]. The 2005 European Consensus on grading of bone marrow fibrosis (ECGMF) [4], included in the 2008 WHO Classification of Tumours of Haematopoietic and Lymphoid Tissues [5] and confirmed, with minor changes, in the 2016 update of WHO classification [6], is the current grading system for bone marrow fibrosis. It focuses mainly on reticulin fibrosis, while collagen deposition and osteosclerosis are not evaluated separately, and lack a well-established grading system.

This article is protected by copyright. All rights reserved. 
The grade of bone marrow fibrosis according to ECGMF (ECGMF grade) bears clinical implications, especially in myeloid neoplasms. It has predictive value on response to therapy and outcome in $B C R-A B L 1$-positive chronic myeloid leukemia [7], and correlates with transfusion requirement and prognosis in myelodysplastic syndromes $[8,9]$. Among BCR-ABL1-negative myeloproliferative neoplasms (MPNs), cases of polycythemia vera with an initial grade of bone marrow fibrosis (MF-1) show an inferior myelofibrosis-free survival, while advanced fibrosis (MF-2 or MF-3) is more frequently associated with a complex karyotype [10,11]. In primary myelofibrosis (PMF), the ECGMF grade is associated with relevant hematological findings, and its prognostic role is proved in comparison with other prognostic scoring systems [12,13]. Moreover, a more accurate stratification of PMF patients is achieved when considering the ECGMF grade along with International Prognostic Scoring System (IPSS) risk categories [14]. Patients with a high ECGMF grade (MF-2 or MF-3) display features indicative of an advanced disease, such as anemia, leukopenia, thrombocytopenia, constitutional symptoms, larger splenomegaly and high IPSS risk categories. They are also more likely to have additional somatic mutations in ASXL1 and EZH2 genes, which are connected to adverse prognosis, and tend to have a significant reduction of overall survival when compared to early PMF patients [15].

Other stromal changes as collagen deposition and osteosclerosis are gaining attention, especially in the context of Janus kinase (JAK) inhibitors therapy [16-23] and hematopoietic stem cell transplantation (HSCT) [24]. Therefore, a careful histological examination is crucial for PMF patients, both at the first biopsy and during followup. A new grading system of bone marrow stromal changes has been recently proposed by Kvasnicka et al., and proved to be reproducible. It evaluates separately reticulin fibrosis, collagen deposition and osteosclerosis, with specific recommendations to improve the staining quality and laboratory standards [25].

In this study, we hypothesize that a comprehensive assessment of reticulin fibrosis, collagen deposition and osteosclerosis may have prognostic implication and allow a better stratification of PMF patients. To verify the hypothesis, reticulin fibrosis, collagen deposition and osteosclerosis were first evaluated separately, according to the grading system proposed by Kvasnicka et al. [25]. Then, for each case, the evaluated stromal changes were summarized in a comprehensive score (RCO score). Finally, the results obtained with RCO score were compared to those obtained with ECGMF grade and IPSS risk categories.

This article is protected by copyright. All rights reserved. 


\section{MATERIALS AND METHODS}

\section{Patients}

The study involved 122 consecutive PMF patients diagnosed between 1998 and 2015 (median follow up: 4.7 years, range: 1.4 months - 13.6 years) at the Hematology Division of the Fondazione IRCCS Ca' Granda - Ospedale Maggiore Policlinico of Milan. Inclusion criteria were the trephine biopsy length (more than $1 \mathrm{~cm}$ ) and the availability of complete clinical history, laboratory and follow-up data, and the patient's informed consent. All cases met the 2008 WHO criteria for PMF, and biopsies were performed at base-line observation: in particular, no patient received prior cytoreductive therapy.

The cases were reviewed by two experienced pathologists (UG, SF) and classified, according to the 2016 updated WHO criteria, as prePMF and overt PMF [6].

\section{Bone Marrow Evaluation}

Formalin-fixed, paraffin-embedded bone marrow trephine biopsies were cut at a 5-micron interval and the resulting sections were stained with Hematoxilin-Eosin, Giemsa, Gomori's silver impregnation and Masson's trichrome. The grade of bone marrow stromal changes was assessed according to the new grading system [25].

We decided not to evaluate microvessel proliferation, another stromal alteration that occurs along with the increase of bone marrow fibrosis [26], since it lacks a well-established grading system.

Perivascular reticulin and collagen fibers were considered as internal quality controls, and fibers density was evaluated only in hematopoietic areas. In case of heterogeneous patterns, the final grade was considered the highest in at least $30 \%$ of bone marrow area.

Reticulin fibrosis was evaluated on Gomori's silver impregnation stained slides and scored as: MF-0, scattered linear reticulin with no intersections (crossovers), corresponding to normal bone marrow; MF-1, loose network of reticulin with many intersections, especially in perivascular areas; MF-2, diffuse and dense increase in reticulin

This article is protected by copyright. All rights reserved. 
with extensive intersections, occasionally with focal bundles of thick fibers mostly consistent with collagen and/or focal osteosclerosis; MF-3, diffuse and dense increase in reticulin with extensive intersections and coarse bundles of thick fibers mostly consistent with collagen, usually associated with osteosclerosis. Collagen deposition was evaluated on Masson's trichrome stained slides and scored as: Co-0, perivascular collagen only (normal); Co-1, focal paratrabecular and/or central collagen deposition without connecting meshwork; Co-2, paratrabecular and/or central deposition of collagen with focally connecting meshwork or generalized paratrabecular apposition of collagen; Co-3, diffuse (complete) connecting meshwork of collagen. Osteosclerosis was evaluated on Hematoxilin-Eosin stained slides and scored as: Ost-0, regular bone trabeculae (distinct paratrabecular borders); Ost-1, focal budding, hooks, spikes or paratrabecular apposition of new bone; Ost-2, diffuse paratrabecular new bone formation with thickening of trabeculae, occasionally with focal interconnections; Ost-3, extensive interconnecting meshwork of new bone with overall effacement of marrow spaces. Examples of the evaluated morphological changes are reported in Figure 1.

A comprehensive score (RCO - Reticulin, Collagen, Osteosclerosis - score), obtained by summing the grade of each morphological parameter (grade of reticulin fibrosis + grade of collagen deposition + grade of osteosclerosis), ranging from 0 to 9 , was assigned to each case. To simplify clinical applicability, the cases were divided into two groups: RCO low-grade (RCO score ranging from 0 to 4) and RCO high-grade (RCO score ranging from 5 to 9). We chose to set a cut-off of 5 for RCO high-grade cases because it implied, in our series, at least two high-grade (grade 2 or 3) stromal changes.

\section{Statistical Analysis}

Continuous variables were expressed as median and range, and categorical variables as absolute frequency and percentage. A logistic model was used to assess association between covariates and mortality. Kaplan-Meier survival analysis was used to evaluate different groups of ECGMF grade, RCO score and IPSS in relation to time.

This article is protected by copyright. All rights reserved. 
Statistical significance was set at a level of $p<0.05$. Sidak correction was used for multiple testing. Given $m$ different null hypotheses and a familywise $\alpha$ of 0.05 , each null hypothesis with a $p$-value lower than $1-(1-\alpha)^{1 / m}$ was rejected.

Data processing and all the statistical analyses were performed using the SAS software (version 9.2; SAS Institute, Cary, NC, USA).

\section{RESULTS}

\section{Base-line characteristics of the patients}

The study involved a consecutive series of 122 PMF patients diagnosed according to the 2008 WHO criteria and revised according to the 2016 update of WHO classification; 69 (57\%) were revised as prePMF (ECGMF grade: MF-0 or MF-1) and 53 (43\%) as overt PMF (ECGMF grade: MF-2 or MF-3).

The series included 56 males and 66 females $(M / F=85 \%)$ with a median age at diagnosis of 68 years (range 30-85). Anemia (hemoglobin $<10 \mathrm{~g} / \mathrm{dL}$ ) was found in 20 patients (16\%), leukocytosis (leukocytes $>25 \times 10 \% / \mathrm{L}$ ) in 4 patients $(3 \%)$, and thrombocytopenia (platelets $<100 \times 10 \%$ ) in 7 patients $(6 \%)$. The main clinical data are summarized in Table 1.

JAK2V617F mutation was detected in 81 cases (66\%). Among the remaining 41, three carried the MPLW515L mutation, one the MPLW515R and 27 a mutation in exon 9 of CALR gene. In the latter group, 15 cases showed a type 1 mutation (del52bp), nine a type 2 mutation (ins5bp), and three other variants (del4bp, del19bp and del5ins12bp). The remaining 10 patients were "triple-negative" (i.e., with no JAK2, CALR or MPL mutations).

According to IPSS, 38 cases were stratified as low risk, 51 as intermediate-1 risk, 21 as intermediate-2 risk, and 12 as high risk.

By the time of the analysis, 21 patients (17\%) had died; leukemic evolution occurred in 14 patients (11.5\%), and thrombo-hemorrhagic events in $25(20.5 \%)$.

This article is protected by copyright. All rights reserved. 


\section{Morphological evaluation of Reticulin, Collagen, Osteosclerosis and RCO score}

The histologic analysis of bone marrow stromal changes, according to the grading system proposed by Kvasnicka et al. [25], gave the following results: reticulin fibrosis, MF-0=9, MF-1=60, MF-2=31 and MF-3=22; collagen deposition, Co-0 $=64$, Co-1=23, Co- $2=21$ and Co-3=14; osteosclerosis, Ost $0=72$, Ost $-1=24$, Ost $-2=19$ and Ost-3=7. Stromal changes evolved harmonically for the three morphological features, with few exceptions. Four out of 60 cases with MF-1 reticulin fibrosis displayed Ost-2 osteosclerosis (7\%) (Figure 2). Moreover, of 31 cases with MF-2 reticulin fibrosis, two were associated with a Co-3 collagen deposition and seven with a Ost-2 osteosclerosis. The details of stromal changes evaluation are listed in Table 2.

Mortality, as a dependent variable in a logistic model, was associated with the grade of reticulin fibrosis and collagen deposition, but not with osteosclerosis. In fact, the risk of death was $3.3\left({ }^{\prime} C_{95 \%} 1.3-9.0\right)$ folds greater in the advanced grade of reticulin fibrosis (grade 2-3 vs 0-1) and 2.7 (I $\mathrm{C}_{95 \%}$ 1.2-6.3) folds greater in the advanced grade of collagen deposition (grade 2-3 vs 0-1).

A comprehensive score (RCO - Reticulin, Collagen, Osteosclerosis - score), obtained by summing the grade of each morphological parameter (grade of reticulin fibrosis + grade of collagen deposition + grade of osteosclerosis), and thus ranging from 0 to 9 , was assigned to each case.

RCO score 1 was the most frequent in our series: it was found in 51 patients $(41.8 \%), 50$ of which showed a MF1/Co-0/Ost-0 combination. RCO score 4 was the second most frequent and found in 14 patients (11.4\%). It was ascribable to a MF-2/Co-1/Ost-1 combination in 11 out of 14 patients $(78.5 \%)$.

RCO scores and individual grades of reticulin fibrosis, collagen deposition and osteosclerosis are reported in Table 3.

This article is protected by copyright. All rights reserved. 


\section{Prognostic significance of RCO score}

To simplify the clinical applicability, we divided the patients in two groups, according to their RCO score: RCO low-grade (RCO score 0-4) and RCO high-grade (RCO score 5-9). Eighty-eight (72\%) out of 122 patients were placed in the RCO low-grade group and $34(28 \%)$ in the RCO high-grade group.

Patients in the RCO high-grade group displayed laboratory features indicative of an advanced disease: they had a higher percentage of anemia, thrombocytopenia, leukocytosis, peripheral blood blasts and increased lactate dehydrogenase (LDH) levels.

Clinical features according to RCO grade group are summarized in Table 4.

RCO score resulted strictly associated $(p<0.001)$ with IPSS risk categories and overall survival (RCO low-grade: 10 dead out of 88 vs RCO high-grade: 11 dead patients out of $34 ; p=0.0059$ ). On the contrary, it was not associated with the percentage of leukemic transformation $(p=0.18)$ or thrombo-hemorrhagic events $(p=0.76)$.

RCO score was able to discriminate the overall survival (OS) of patients: the $75^{\text {th }}$ percentile OS in the RCO score low-grade group was 12.5 years, against 3.5 years in the RCO score high-grade group (Log-Rank test: $p<0.001)$.

We compared the OS curves obtained with RCO score to those obtained with ECGMF grade: RCO score turned out to be more accurate in identifying high risk patients with poor prognosis (Figure $3 \mathrm{~A}$ ).

The RCO low-grade group included 11 overt PMF patients (MF-2) with RCO score 4. These patients did not seem to differ in terms of OS when compared to prePMF (MF-0 or MF-1) RCO low-grade patients (Figure 3A). We compared clinical and pathological features of prePMF and overt PMF patients in the RCO low-grade group. As seen in Table 5, no significant difference was found.

We matched the OS curves obtained with RCO score to those obtained with IPSS risk categories: the two systems showed comparable results, even if IPSS was slightly better in differentiating high risk patients after 60 months of follow-up (Figure 3B).

Then we investigated if a combined evaluation of RCO score and IPSS risk categories could allow a more accurate stratification. For this purpose, each patient was assigned to a further risk subgroup, created by an

This article is protected by copyright. All rights reserved. 
integrated estimation of IPSS risk categories and RCO score: Low-risk (IPSS low/intermediate-1 risk + RCO lowgrade), Moderate-risk (IPSS intermediate-2/high-risk + RCO low-grade or IPSS low/intermediate-1 risk + RCO high-grade), and High-risk (IPSS intermediate-2/high risk + RCO high-grade). RCO score proved to add prognostic information to IPSS risk categories: in fact, the combined evaluation was more accurate than IPSS alone. For instance, the median survival of intermediate-2/high-risk IPSS patients declined from 9.7 years to 4.9 years if a high-grade RCO score was associated. A significant difference was also found in OS curves obtained with RCO score and IPSS together $(p<0.001)$. These results are summarized in Figure $3 \mathrm{C}$.

The combined evaluation provided an increase in positive predictive value (PPV) for mortality in the High-risk group: PPV raised from 32\% (when considering RCO score alone) and 36\% (when considering IPSS alone), to $42 \%$ when considering RCO score and IPSS together. The results are listed in Table 6.

Finally, we compared OS curves resulting from two different combined analyses: IPSS + RCO score vs IPSS + ECGMF grade. The former combination was more accurate in discriminating the OS of High-risk patients, especially after 60 months of follow-up (Figure 3D).

\section{DISCUSSION}

We hypothesized that a comprehensive histologic evaluation of reticulin fibrosis, collagen deposition and osteosclerosis, according to the grading system proposed by Kvasnicka et al. [25] may have prognostic implication and may allow a better stratification of PMF patients.

To verify the hypothesis, we assessed reticulin fibrosis, collagen deposition and osteosclerosis in base-line bone marrow trephine biopsies of 122 consecutive PMF patients, assigning a grade from 0 to 3 to each parameter. A comprehensive score (RCO score, obtained by summing the individual grade of Reticulin fibrosis, Collagen deposition and Osteosclerosis) for each case enabled us to identify two groups of patients characterized by lowgrade (RCO score 0-4) and high-grade (RCO score 5-9) bone marrow stromal changes, and by different clinical features. In fact, patients in the RCO high-grade group more frequently displayed laboratory features indicative of an advanced disease, such as anemia, thrombocytopenia, peripheral blood blasts and increased LDH levels.

This article is protected by copyright. All rights reserved. 
RCO score resulted strictly associated with OS and IPSS risk categories. OS curves according to RCO score were significantly different for low- and high-grade groups: the $75^{\text {th }}$ percentile value of 12.5 years for RCO lowgrade patients decreased to 3.5 years for RCO high-grade patients. Moreover, RCO score resulted more accurate than the current grading system, based on the ECGMF grade, in identifying high risk patients with poor prognosis. Interestingly, the subset of RCO low-grade patients with overt PMF (ECGMF grade MF-2) according to the 2016 update of WHO classification displayed an OS curve comparable to RCO low-grade patients with prePMF (ECGMF grade MF-0 or MF-1), and did not show any significant clinical and pathological differences. This finding furtherly supports the value of a comprehensive histologic evaluation of bone marrow stromal changes in stratifying PMF patients.

Finally, a combined assessment of RCO score and IPSS risk categories increased PPV for mortality. In fact, for High-risk patients (RCO high-grade and IPSS intermediate-2/high risk), PPV raised from 32\% (when considering RCO score alone) and 36\% (when considering IPSS alone), to $42 \%$ when considering RCO score and IPSS together. This means that the application of the RCO along with the IPSS risk category allows a better stratification of PMF patients.

To our knowledge, this is the first study that specifically investigates the prognostic significance of a comprehensive histologic evaluation of reticulin fibrosis, collagen deposition and osteosclerosis in a series of PMF patients.

The ECGMF grade is known to play a major prognostic role in PMF patients. For example, when our group examined the prognostic significance of ECGMF grade in PMF patients and its relationship with IPSS risk categories $[13,14]$, the two systems resulted independently able to predict survival at a multivariate analysis. Additionally, a more accurate prognostication was achieved when the two systems were used together. More recently, a work by Guglielmelli et al. [15] on a large series of PMF patients confirmed our results, disclosing that a high ECGMF grade (MF-2 or MF-3) is associated with cytopenias, circulating blasts, larger splenomegaly and a higher IPSS risk category. These patients were also enriched in prognostically unfavorable mutations, in particular ASXL1 and EZH2. These data suggest that a high ECGMF grade represents an adverse prognostic

This article is protected by copyright. All rights reserved. 
factor, and that it can provide supplementary information in lower IPSS risk categories, a group of patients where clear indications for treatment are still missing.

Other stromal changes, as collagen deposition and osteosclerosis, remained under-recognized, although commonly found in association with advanced bone marrow fibrosis in $B C R$-ABL1-negative MPNs $[27,28]$. A grading system for reticulin fibrosis, collagen deposition and osteosclerosis was recently proposed by Kvasnicka et al. [25]. It proved to be reproducible in a multicenter study that involved 11 haematopathologists in a blinded assessment. The overall inter-rater reliability ranged between 0.898 and 0.926 for all three analyzed parameters (reticulin fibrosis, collagen deposition and osteosclerosis). An additional issue of that work consists in the identification of specific problems and pitfalls in the interpretation of each single morphological parameter (for instance, biopsy length, presence of crushing artefacts and squeezing, thickness of sections and quality of staining).

Collagen deposition and osteosclerosis are gaining attention as indicators of therapy response, in the background of JAK inhibitors treatment and HSCT. In fact, a stabilization or regression of reticulin fibrosis, collagen deposition and osteosclerosis is described after prolonged therapy with JAK inhibitors [18-23]. While a prompt reduction in reticulin fibrosis is also reported after HSCT, the complete regression of collagen deposition or osteosclerosis is unclear [24]. Hence, a reproducible grading system for collagen deposition and osteosclerosis is necessary for a supervision of response to therapy in these patients.

In conclusion, our study documents that the histologic evaluation of reticulin fibrosis, collagen deposition and osteosclerosis in a comprehensive RCO score is prognostically significant, more accurate than ECGMF grade in identifying high risk patients with poor prognosis, and improves PPV for mortality when applied in addition to IPSS risk categories. Still, two important limitations of our study consist in its retrospective nature and the relatively low number of examined patients and clinical events. For this reason, prospective studies with a larger number of patients are needed to confirm the prognostic value of the RCO score, to evaluate its relationship with the presence of adverse somatic mutations, such as ASXL1 and EZH2, and its relevance in management of PMF patients at diagnosis and after therapy.

This article is protected by copyright. All rights reserved. 


\section{Authors' contributions}

UG was responsible for the integrity of the work as a whole.

UG, SF, DC and Al designed the study, analyzed the data and wrote the manuscript.

$A B$ and IC performed statistical analyses.

UG, SF, ArBo and GE revised bone marrow biopsies.

Al, DC, CB and NO provided clinical data and followed the patients.

GB revised the paper.

UG and $\mathrm{Al}$ approved the final version of the manuscript.

\section{Ethics statement}

This study has been performed according to the Declaration of Helsinki.

\section{REFERENCES}

1. Kuter DJ, Bain B, Mufti G, Bagg A, Hasserjian RP. Bone marrow fibrosis: pathophysiology and clinical significance of increased bone marrow stromal fibres. Br J Haematol 2007; 139: 351-362.

2. Kreipe $H$, Büsche $G$, Bock $O$ et al. Myelofibrosis: molecular and cell biological aspects. Fibrogenesis Tissue Repair 2012; 5(Suppl 1): S21.

3. Bauermeister DE. Quantitation of bone marrow reticulin--a normal range. Am J Clin Pathol 1971; 56: 2431.

This article is protected by copyright. All rights reserved. 
4. Thiele J, Kvasnicka HM, Facchetti F, Franco V, van der Walt J, Orazi A. European Consensus on grading of bone marrow fibrosis and assessment of cellularity. Haematologica 2005; 90: 1128-1132.

5. Swerdlow SH, Campo E, Harris NL et al., eds. World Health Organization Classification of Tumours of Haematopoietic and Lymphoid Tissues. Lyon, France: IARC Press; 2008.

6. Arber DA, Orazi A, Hasserjian R et al. The 2016 revision to the World Health Organization classification of myeloid neoplasms and acute leukemia. Blood 2016; 127: 2391-2405.

7. Kvasnicka HM, Thiele J, Schmitt-Graeff A et al. Prognostic impact of bone marrow erythropoietic precursor cells and myelofibrosis at diagnosis of $\mathrm{Ph} 1+$ chronic myelogenous leukaemia--a multicentre study on 495 patients. Br J Haematol 2001; 112: 727-739.

8. Della Porta MG, Malcovati L, Boveri E et al. Clinical relevance of bone marrow fibrosis and CD34positive cell clusters in primary myelodysplastic syndromes. J Clin Oncol 2009; 27: 754-762.

9. Fu B, Jaso JM, Sargent RL et al. Bone marrow fibrosis in patients with primary myelodysplastic syndromes has prognostic value using current therapies and new risk stratification systems. Mod Pathol $2014 ; 27: 681-689$

10. Barbui $\mathrm{T}$, Thiele $\mathrm{J}$, Passamonti $\mathrm{F}$ et al. Initial bone marrow reticulin fibrosis in polycythemia vera exerts an impact on clinical outcome. Blood 2012; 119: 2239-2241.

11. Boiocchi L, Mathew S, Gianelli $U$ et al. Morphologic and cytogenetic differences between postpolycythemic myelofibrosis and primary myelofibrosis in fibrotic stage. Mod Pathol 2013; 26: $1577-$ 1585.

12. Thiele J, Kvasnicka HM. Grade of bone marrow fibrosis is associated with relevant haematological findings-a clinicopathological study on 865 patients with chronic idiopathic myelofibrosis. Ann Hematol 2006; 85: 226-232.

13. Vener C, Fracchiolla NS, Gianelli U et al. Prognostic implications of the European Consensus for grading bone marrow fibrosis in chronic idiopathic myelofibrosis. Blood 2008; 111: 1862-1865.

This article is protected by copyright. All rights reserved. 
14. Gianelli U, Vener C, Bossi A et al. The European Consensus on grading of bone marrow fibrosis allows a better prognostication of patients with primary myelofibrosis. Mod Pathol 2012; 25: 1193-1202.

15. Guglielmelli $P$, Rotunno $G$, Pacilli $A$ et al. Prognostic impact of bone marrow fibrosis in primary myelofibrosis. A study of the AGIMM group on 490 patients. Am J Hematol 2016; 91: 918-922.

16. Kroeger $\mathrm{N}$, Thiele $\mathrm{J}$, Zander $\mathrm{A}$ et al. Rapid regression of bone marrow fibrosis after dose-reduced allogeneic stem cell transplantation in patients with primary myelofibrosis. Exp Hematol 2007; 35: 17191722.

17. Thiele J, Kvasnicka HM, Dietrich $\mathrm{H}$ et al. Dynamics of bone marrow changes in patients with chronic idiopathic myelofibrosis following allogeneic stem cell transplantation. Histol Histopathol 2005; 20: 879_ 889.

18. Verstovsek S, Kantarjian HM, Estrov Z et al. Long-term outcomes of 107 patients with myelofibrosis receiving JAK1/JAK2 inhibitor ruxolitinib: survival advantage in comparison to matched historical controls. Blood 2012; 120; 1202-1209.

19. Kvasnicka HM, Thiele J, Bueso-Ramos CE et al. Long term effects of ruxolitinib therapy on bone marrow morphology in patients with myelofibrosis in comparison with best available therapy. Haematologica 2013; 98(Suppl 1): S591.

20. Kvasnicka HM, Thiele J, Bueso-Ramos CE et al. Effects of five years of ruxolitinib therapy on bone marrow morphology in patients with myelofibrosis and comparison with best available therapy. Blood 2013; $122: 4055$.

21. Wilkins BS, Radia D, Woodley C, Farhi SE, Keohane C, Harrison CN. Resolution of bone marrow fibrosis in a patient receiving JAK1/JAK2 inhibitor treatment with ruxolitinib. Haematologica 2013; 98: 1872-1876.

22. Iurlo A, Gianelli U, Rapezzi D et al. Imatinib and ruxolitinib association: first experience in two patients. Haematologica 2014; 99: e76-e77.

This article is protected by copyright. All rights reserved. 
23. Iurlo $A$, Cattaneo $D$, Boiocchi $L$ et al. Clinical and morphologic features in five post-polycythemic myelofibrosis patients treated with ruxolitinib. Ann Hematol 2015; 94: 1749-1751.

24. Tamari R, Mughal TI, Rondelli D et al. Allo-SCT for myelofibrosis: reversing the chronic phase in the JAK inhibitor era? Bone Marrow Transplantation 2015; 50: 628-636.

25. Kvasnicka HM, Beham-Schmid C, Bob R et al. Problems and pitfalls in grading of bone marrow fibrosis, collagen deposition and osteosclerosis - a consensus-based study. Histopathology 2016; 68: 905-915.

26. Mesa RA, Hanson CA, Rajkumar VS, Schroeder G, Tefferi A. Evaluation and clinical correlations of bone marrow angiogenesis in myelofibrosis with myeloid metaplasia. Blood 2000; 96: 3374-3380.

27. Bock O, Loch G, Schade $U$ et al. Osteosclerosis in advanced chronic idiopathic myelofibrosis is associated with endothelial overexpression of osteoprotegerin. Br J Haematol 2005; 130: 76-82.

28. Poulsen LW, Melsen F, Bendix K. A histomorphometric study of haematological disorders with respect to marrow fibrosis and osteosclerosis. APMIS 1998; 106: 495-499.

This article is protected by copyright. All rights reserved. 


\section{FIGURES LEGEND}

Figure 1. Examples of different grades of bone marrow stromal changes: first row, reticulin fibrosis (MF-0, MF-1, MF-2, and MF-3; Gomori's silver impregnation, 20x); second row, collagen deposition (Co-0, Co-1, Co-2, and Co3; Masson's trichrome staining, 20x); third row, osteosclerosis (Ost-0, Ost-1, Ost-2, and Ost-3; Hematoxilin-Eosin staining, 20x).

Figure 2. Example of a case with discordant stromal features. A loose interconnecting reticulin meshwork, grade MF-1 (A, Gomori's silver impregnation, 20x) was associated with focal paratrabecular collagen fibers, grade Co-1 (B, Masson's trichrome, 20x) and with a diffuse thickening of bone trabeculae, with hooks, spikes and focal interconnections, grade Ost-2 (C, Hematoxilin-eosin, 10x). The case was included in low-grade RCO score group $(\mathrm{RCO}$ score $=4)$.

Figure 3A. Overall survival curves of the $122 \mathrm{PMF}$ patients stratified according to the ECGMF grade (grey line) vs the RCO score (black line). RCO low-grade (score 0-4) and RCO high-grade (score 5-9).

Figure 3B. Overall survival curves of the 122 PMF patients stratified according to IPSS (grey line) vs RCO score (black line). RCO low-grade (score 0-4) and RCO high-grade (score 5-9).

Figure 3C. Overall survival curves of the 122 PMF patients stratified according to IPSS alone (grey lines) vs IPSS + RCO (black lines). Low-risk (IPSS low/intermediate-1 risk + RCO low-grade), Moderate-risk (IPSS intermediate-2/high risk + RCO low-grade or IPSS low/intermediate-1 risk + RCO highgrade) and High-risk (IPSS intermediate-2/high risk + RCO high-grade score).

Figure 3D. Overall survival curves of the $122 \mathrm{PMF}$ patients stratified according to the combined evaluation of IPSS + ECGMF grade (grey lines) vs IPSS + RCO (black lines). IPSS + ECGMF: Low-risk (IPSS low/intermediate-1 risk + ECGMF MF-0/MF-1), Moderate-risk (IPSS low/intermediate-1 risk + ECGMF: MF-2/MF-3 or IPSS intermediate-2/high risk + ECGMF MF-0/MF-1) and High-risk (IPSS: intermediate-2/high risk + ECGMF MF-2/MF-3).

IPSS + RCO: Low-risk (IPSS low/intermediate-1 risk + RCO low-grade), Moderate-risk (IPSS intermediate-2/high risk + RCO low-grade or IPSS low/intermediate-1 risk + RCO high-grade) and High-risk (IPSS intermediate-2/high risk + RCO high-grade).

This article is protected by copyright. All rights reserved. 
Table 1. Base-line clinical features of the $122 \mathrm{PMF}$ patients according to the 2016 update of WHO classification.

\begin{tabular}{|c|c|c|c|c|}
\hline & prePMF & Overt PMF & Totals & $p$ value $(* *)$ \\
\hline No. of patients (\%) & $69(56.6)$ & $53(43.4)$ & 122 & \\
\hline Median age at diagnosis, years (range) & $66(30-84)$ & $69(37-85)$ & $68(30-85)$ & 0.058 \\
\hline $\mathrm{M} / \mathrm{F}$, ratio & $31 / 38(0.82)$ & $25 / 28(0.89)$ & $56 / 66(0.85)$ & 0.805 \\
\hline $\mathrm{Hb}$ level (g/dL), median (range) & $13.7(6.8-17.6)$ & $11.5(8.1-15.9)$ & $12.8(6.8-17.6)$ & 0.0001 \\
\hline $\mathrm{Hb}$ level $<10 \mathrm{~g} / \mathrm{dL}$, no. of patients (\%) & $5(7.2)$ & $15(28.3)$ & $20(16.3)$ & 0.0018 \\
\hline PLT count (x109/L), median (range) & $726(54-1695)$ & $424(38-1685)$ & $681(38-1695)$ & $<0.0001$ \\
\hline PLT count $<100 \times 10 \%$, no. of patients (\%) & $1(1.4)$ & $6(11.3)$ & $7(5.7)$ & 0.042 \\
\hline WBC count (x109/L), median (range) & $9(3.3-22.1)$ & $10.3(1.9-64.3)$ & $9.5(1.9-64.3)$ & 0.057 \\
\hline WBC count $>25 \times 10 \%$, no. of patients (\%) & 0 & $4(7.5)$ & $4(3.3)$ & 0.033 \\
\hline Circulating blasts $\geq 1 \%$, no. of patients (\%) & $1(1.5)$ & $19(35.8)$ & $20(16.4)$ & $<0.0001$ \\
\hline LDH (IU/l)*, median (range) & $441(172-1293)$ & $853(174-2640)$ & $518(172-2640)$ & $<0.0001$ \\
\hline No. of deaths (\%) & $6(8.7)$ & $15(28.3)$ & $21(17.2)$ & 0.005 \\
\hline No. of leukemic evolutions (\%) & $5(7.2)$ & $9(17.0)$ & $14(11.5)$ & 0.150 \\
\hline No. of thrombo-hemorrhagic events (\%) & $13(18.8)$ & $12(22.6)$ & $25(20.5)$ & 0.655 \\
\hline JAK2 mutated, no. of patients (\%) & $51(73.9)$ & $30(56.6)$ & 81 (66.3) & 0.045 \\
\hline CALR mutated, no. of patients (\%) & $13(18.8)$ & $14(26.4)$ & $27(22.1)$ & 0.045 \\
\hline Type 1 mutation & $6(8.7)$ & $9(16.9)$ & $15(12.3)$ & \\
\hline Type 2 mutation & $5(7.2)$ & $4(7.5)$ & $9(7.4)$ & \\
\hline Other mutations & $2(2.9)$ & $1(1.9)$ & $3(2.5)$ & \\
\hline$M P L$ mutated, no. of patients (\%) & $1(1.4)$ & $3(5.7)$ & $4(3.3)$ & 0.316 \\
\hline Triple negative, no. of patients (\%) & $4(5.8)$ & $6(11.3)$ & $10(8.2)$ & 0.270 \\
\hline \multicolumn{5}{|l|}{ IPSS, no. of patients (\%) } \\
\hline Low risk & $31(44.9)$ & $7(13.2)$ & $38(31.1)$ & \multirow{4}{*}{$<0.0001$} \\
\hline Intermediate-1 risk & $34(49.3)$ & $17(32.1)$ & $51(41.8)$ & \\
\hline Intermediate-2 risk & $3(4.3)$ & $18(34.0)$ & $21(17.2)$ & \\
\hline High risk & $1(1.4)$ & $11(20.7)$ & $12(9.8)$ & \\
\hline
\end{tabular}

$\left({ }^{\star}\right)$ LDH normal values: $135-214$ IU/l.

$\left.{ }^{* \star}\right)$ Given $\mathrm{m}(=18)$ different null hypotheses and a familywise $\alpha$ of 0.05 , each null hypotheses that has a $p$-value lower than $1-(1-\alpha)^{1 / m}(=0.0028)$ is rejected.

This article is protected by copyright. All rights reserved. 
Table 2. Details of stromal changes evaluation in 122 PMF patients.

\begin{tabular}{|l|r|r|r|r|r|r|r|r|r|}
\hline & & \multicolumn{4}{|c|}{ Collagen deposition } & \multicolumn{3}{c|}{ Osteosclerosis } \\
\hline Reticulin fibrosis & Totals & Co-0 & Co-1 & Co-2 & Co-3 & Ost-0 & Ost-1 & Ost-2 & Ost-3 \\
\hline MF-0 & $\mathbf{9}$ & 9 & 0 & 0 & 0 & 8 & 1 & 0 & 0 \\
\hline MF-1 & $\mathbf{6 0}$ & 54 & 6 & 0 & 0 & 56 & 0 & 4 & 0 \\
\hline MF-2 & 31 & 0 & 17 & 12 & $\mathbf{2}$ & 8 & 16 & 7 & 0 \\
\hline MF-3 & $\mathbf{2 2}$ & 1 & 0 & 9 & 12 & 0 & 7 & 8 & 7 \\
\hline Totals & 122 & $\mathbf{6 4}$ & $\mathbf{2 3}$ & $\mathbf{2 1}$ & 14 & $\mathbf{7 2}$ & $\mathbf{2 4}$ & 19 & $\mathbf{7}$ \\
\hline
\end{tabular}

Table 3. Frequency distribution of RCO scores and percentage of patients with high-grade (grade 2 or 3 ) reticulin fibrosis, collagen deposition and osteosclerosis.

\begin{tabular}{|r|r|r|r|r|r|r|r|r|r|r|}
\cline { 2 - 10 } \multicolumn{1}{r|}{ RCO score } & $\mathbf{0}$ & $\mathbf{1}$ & $\mathbf{2}$ & $\mathbf{3}$ & $\mathbf{4}$ & $\mathbf{5}$ & $\mathbf{6}$ & $\mathbf{7}$ & $\mathbf{8}$ & $\mathbf{9}$ \\
\hline Frequency & 8 & 51 & 6 & 9 & 14 & 7 & 8 & 9 & 4 & 6 \\
\hline MF-2 or MF-3 (\%) & & & & 55.6 & 100.0 & 100.0 & 100.0 & 100.0 & 100.0 & 100.0 \\
\hline Co-2 or Co-3 (\%) & & & & & 21.5 & 71.4 & 100.0 & 100.0 & 100.0 & 100.0 \\
\hline Ost-2 or Ost-3 (\%) & & & & & & 28.6 & 50.0 & 66.7 & 100.0 & 100.0 \\
\hline
\end{tabular}

This article is protected by copyright. All rights reserved. 
Table 4. Base-line clinical features of the 122 PMF patients according to RCO score.

RCO score

\begin{tabular}{|c|c|c|c|}
\hline & Low-grade & High-grade & $p$ value $(* *)$ \\
\hline No. of patients (\%) & $88(72.1)$ & $34(27.9)$ & \\
\hline Median age at diagnosis, years (range) & $68(30-85)$ & $69(37-85)$ & 0.147 \\
\hline $\mathrm{M} / \mathrm{F}$, ratio & $38 / 50(0.76)$ & $18 / 16(1.13)$ & 0.332 \\
\hline Hb level (g/dL), median (range) & $13.3(6.8-17.6)$ & $10.7(8.1-15.0)$ & $<0.0001$ \\
\hline $\mathrm{Hb}$ level $<10 \mathrm{~g} / \mathrm{dL}$, no. of patients (\%) & $7(8.0)$ & $13(38.2)$ & $<0.0001$ \\
\hline PLT count (x109/L), median (range) & $715(54-1695)$ & $303(38-1129)$ & $<0.0001$ \\
\hline PLT count $<100 \times 10^{9} / \mathrm{L}$, no. of patients (\%) & $1(1.14)$ & $6(17.6)$ & 0.0018 \\
\hline WBC count ( $\left.\times 10^{9} / \mathrm{L}\right)$, median (range) & $9.4(3.3-22.1)$ & $10.7(1.9-64.3)$ & 0.154 \\
\hline WBC count $>25 \times 10 \% / L$, no. of patients (\%) & $0(0.0)$ & $4(11.8)$ & 0.0053 \\
\hline Circulating blasts $\geq 1 \%$, no. of patients (\%) & $3(3.4)$ & $17(50.0)$ & $<0.0001$ \\
\hline LDH $(\mathrm{IU} / \mathrm{l})^{*}$, median (range) & $466(172-1879)$ & $907(174-2640)$ & $<0.0001$ \\
\hline No. of deaths (\%) & $10(11.4)$ & $11(32.4)$ & 0.0059 \\
\hline No. of leukemic evolutions (\%) & $8(9.1)$ & $6(17.6)$ & 0.184 \\
\hline No. of thrombo-hemorrhagic events (\%) & $21(23.9)$ & $9(26.5)$ & 0.764 \\
\hline JAK2 mutated, no. of patients (\%) & $61(69.3)$ & $20(58.8)$ & 0.271 \\
\hline \multicolumn{4}{|l|}{ IPSS, no. of patients (\%) } \\
\hline Low risk & $35(39.8)$ & $3(8.8)$ & \multirow{4}{*}{$<0.0001$} \\
\hline Intermediate-1 risk & $44(50.0)$ & $7(20.6)$ & \\
\hline Intermediate-2 risk & $8(9.1)$ & $13(38.2)$ & \\
\hline High risk & $1(1.1)$ & $11(32.4)$ & \\
\hline
\end{tabular}

$\left(^{*}\right) \mathrm{LDH}$ normal values: $135-214 \mathrm{IU} / \mathrm{l}$.

$\left({ }^{* *}\right)$ Given $m(=15)$ different null hypotheses and a familywise $\alpha$ of 0.05 , each null hypothesis with a $p$-value lower than $1-(1-\alpha)^{1 / m}(=0.0034)$

is rejected.

This article is protected by copyright. All rights reserved. 
Table 5. Base-line clinical features of the 88 RCO low-grade patients according to the grade of reticulin fibrosis (ECGMF grade).

\begin{tabular}{|c|c|c|c|}
\hline \multicolumn{4}{|c|}{ Low-grade RCO score group } \\
\hline & MF-0 + MF-1 & MF-2 + MF-3 & $p$ value $(* *)$ \\
\hline No. of patients (\%) & $69(78)$ & $19(22)$ & \\
\hline Median age at diagnosis, years (range) & $68(35-84)$ & $70(39-89)$ & 0.2425 \\
\hline$M / F$, ratio & $31 / 38(0.82)$ & $7 / 12(0.58)$ & 0.607 \\
\hline $\mathrm{Hb}$ level (g/dL), median (range) & $12.8(6.8-17.6)$ & $13.1(9.5-15.9)$ & 0.2781 \\
\hline $\mathrm{Hb}$ level < $10 \mathrm{~g} / \mathrm{dL}$, no. of patients (\%) & $5(7)$ & $2(10)$ & 0.641 \\
\hline PLT count (x109/L), median (range) & $681(54-1695)$ & $674(116-1685)$ & 0.3801 \\
\hline PLT count $<100 \times 10 \%$, no. of patients (\%) & $1(1)$ & $0(0.0)$ & 1.000 \\
\hline WBC count (x109/L), median (range) & $9.5(3.3-22.1)$ & $9.4(5.55-20.7)$ & 0.2798 \\
\hline WBC count $>25 \times 10 \% / L$, no. of patients (\%) & $0(0.0)$ & $0(0.0)$ & 1.000 \\
\hline Circulating blasts $\geq 1 \%$, no. of patients (\%) & $1(1)$ & $2(10)$ & 0.116 \\
\hline LDH $(\mathrm{IU} /)^{*}$, median (range) & $518(172-1293)$ & $533(264-1879)$ & 0.0034 \\
\hline No. of deaths (\%) & $6(9)$ & $4(21)$ & 0.213 \\
\hline No. of leukemic evolutions (\%) & $5(8)$ & $3(16)$ & 0.362 \\
\hline No. of thrombo-hemorrhagic events (\%) & $16(23)$ & $5(26)$ & 0.768 \\
\hline JAK2 mutated, no. of patients (\%) & $51(74)$ & $10(52)$ & 0.095 \\
\hline \multicolumn{4}{|l|}{ IPSS, no. of patients (\%) } \\
\hline Low risk & $31(45)$ & $4(21)$ & 0.069 \\
\hline Intermediate-1 risk & $34(49)$ & $10(53)$ & \\
\hline Intermediate-2 risk & $3(4)$ & $5(26)$ & \\
\hline High risk & $1(1)$ & $0(0.0)$ & \\
\hline
\end{tabular}

\section{(*) LDH normal values: 135-214 IU/l.}

$\left.{ }^{* \star}\right)$ Given $\mathrm{m}(=15)$ different null hypotheses and a familywise $\alpha$ of 0.05 , each null hypothesis with a $p$-value lower than $1-(1-\alpha)^{1 / m}(=0.0034)$ is rejected.

This article is protected by copyright. All rights reserved. 
Table 6. Absolute mortality (and percentage) in relation to risk subgroups obtained with a combined evaluation of IPSS risk categories + RCO score.

\begin{tabular}{|l|c|c|c|c|c|}
\hline & \multicolumn{2}{|c|}{ Dead } & \multicolumn{3}{c|}{ Alive** $^{*}$} \\
\hline Risk subgroup* & $\mathbf{n}$ & $\%$ & $\mathbf{n}$ & $\%$ & Total \\
\hline Low-risk & 8 & 10.0 & 71 & 89.9 & 79 \\
\hline Moderate-risk & 3 & 15.8 & 16 & 84.2 & 19 \\
\hline High-risk & 10 & 41.7 & 14 & 58.3 & 24 \\
\hline Total & 21 & 17.2 & 101 & 82.8 & 122 \\
\hline
\end{tabular}

${ }^{(*)}$ Low-risk (IPSS low/intermediate-1 risk + RCO low-grade), Moderate-risk (IPSS intermediate-2/high risk + RCO low-grade or IPSS low/intermediate-1 risk + RCO high-grade) and High-risk (IPSS intermediate-2/high risk + RCO high-grade score).

$\left.{ }^{* *}\right)$ Alive or lost at follow-up.

This article is protected by copyright. All rights reserved. 


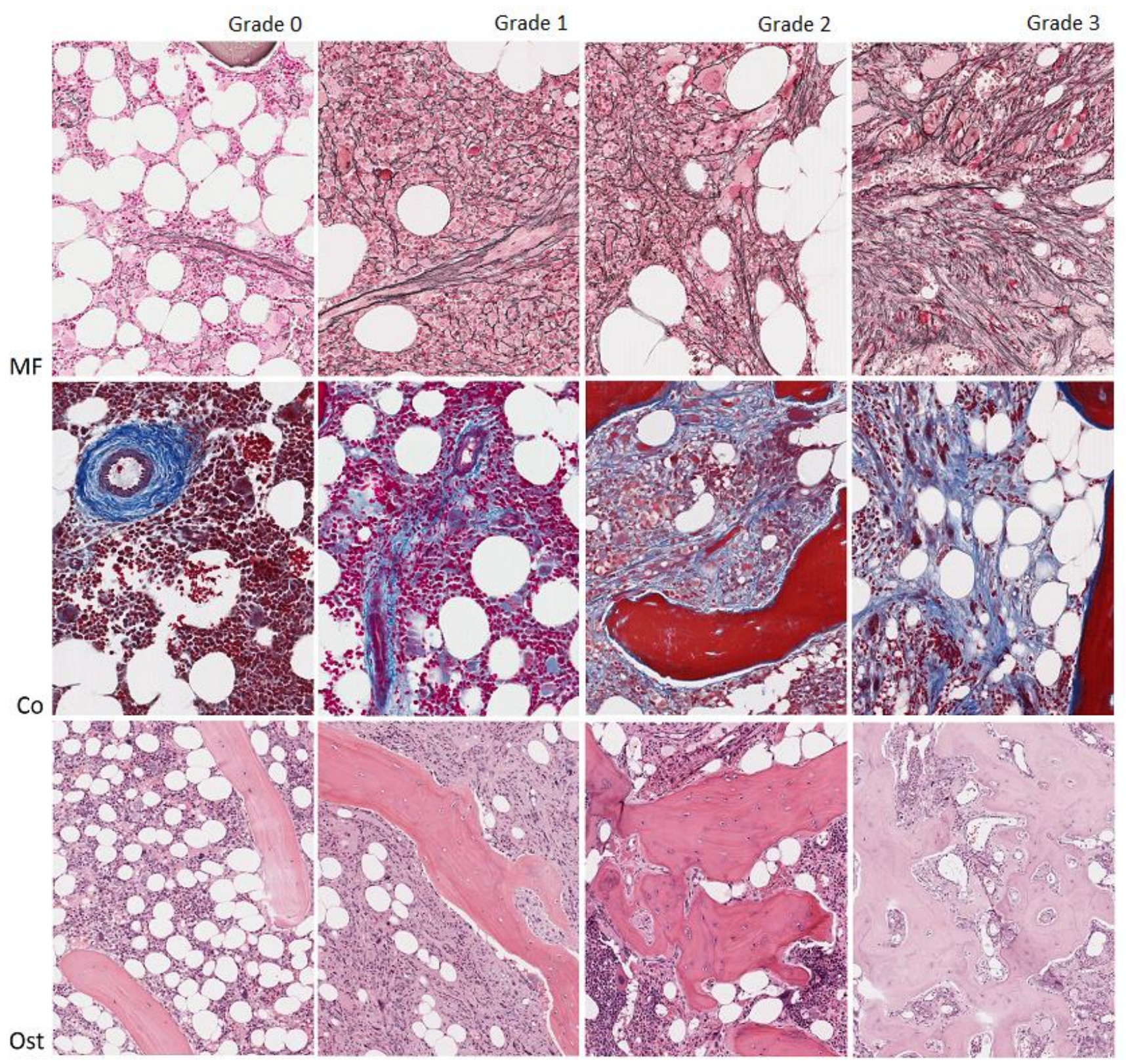

This article is protected by copyright. All rights reserved. 

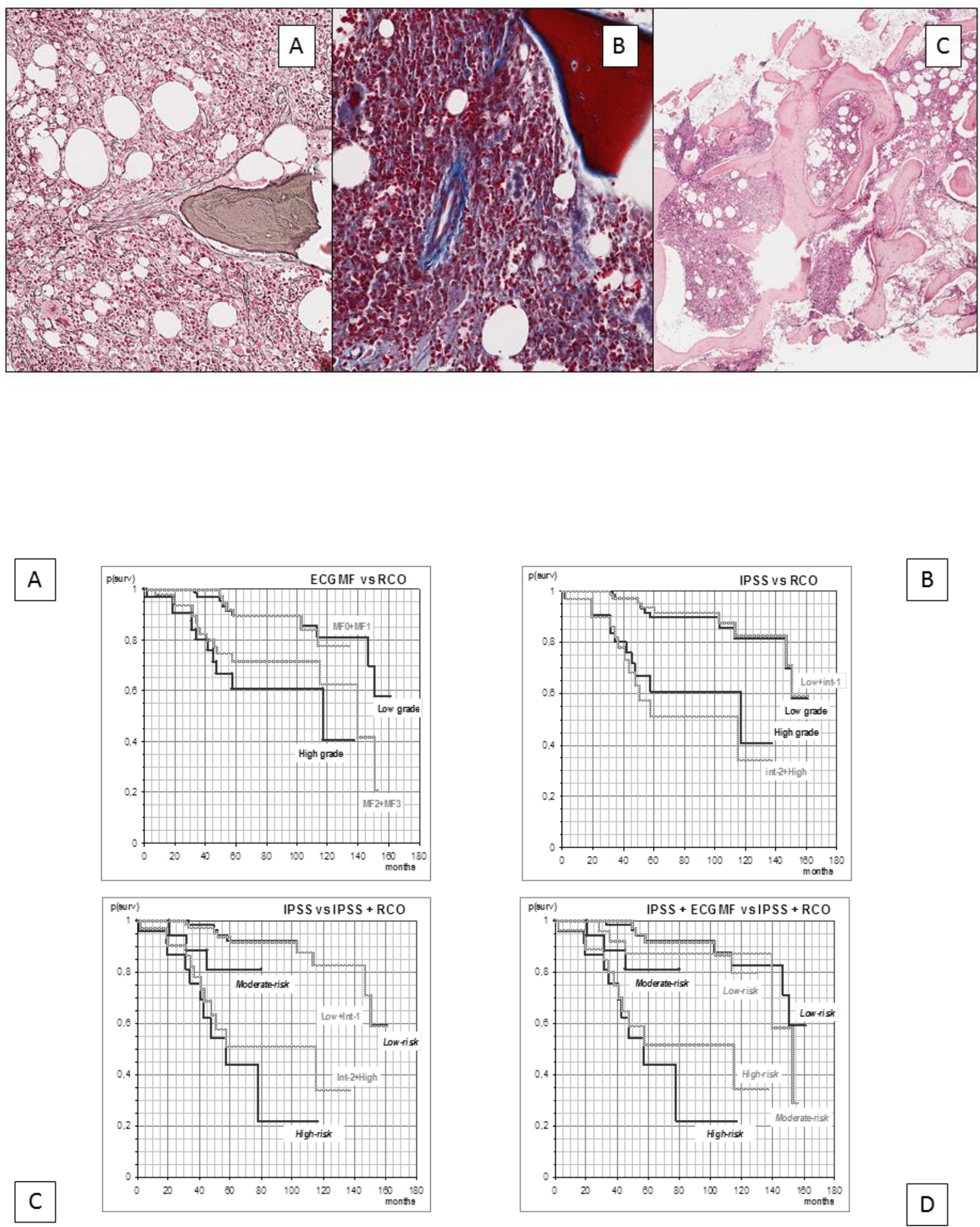

This article is protected by copyright. All rights reserved. 